\section{Do endurance sports affect female hearts differently to male hearts?}

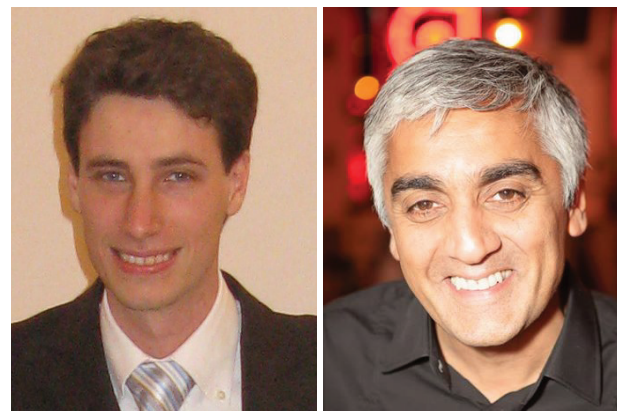

Gherardo Finocchiaro ${ }^{1} \&$ Sanjay Sharma ${ }^{*, 1}$
"In an era where the number of females participating in

endurance events is

increasing...it is prudent to have some understanding of the impact of intensive exercise on the female heart."
First draft submitted: 25 November 2015; Accepted for publication: 8 December 2015; Published online: 26 February 2016
Long-term athletic training is associated with a series of alterations in cardiac structure and function that permit the generation of a large stroke volume required to deliver an increased cardiac output for a prolonged period $[1,2]$. The magnitude of these physiological adaptations is governed by several factors including the sex of the athlete [3].

For many years competitive sport has been dominated by males, but the last two decades have witnessed an increasing number of women participating in high-level competition and female athletes comprised almost $45 \%$ of all athletes at the London 2012 Olympics [4]. Females also excel in sporting disciplines that were traditionally considered to represent purely male sports, such as soccer, rugby and boxing. Despite these observations, the majority of studies investigating the physiological effects of exercise on the heart are focused on male athletes, with few reports on female counterparts [5]. Although there is only a single chromosome separating the two sexes, females differ from males in several respects including their anthropometric, biochemical, physiological and psychological profile, all of which play a role in their cardiovascular response to athletic training. In general, females are smaller, have lower testosterone concentrations [6] and lower lean body mass, therefore they cannot achieve the same workload compared to male counterparts of equivalent size and standard. Various studies in small cohorts of athletes have shown a significant sex difference in left ventricular (LV) mass corrected for bodyweight [7]. The higher LV mass in males is considered an important factor for the higher $\mathrm{VO}^{2}$ max observed in male compared to female athletes [8].

\section{The athlete's heart}

Both males and females exhibit similar qualitative adaptive changes in the heart compared to sedentary controls. Irrespective of sex, athletes generally show a $10-20 \%$ increase in LV wall thickness and a $10 \%$ increase in LV size compared to controls. There is also a concomitant
'Cardiovascular Sciences Research Centre, St George's, University of London, Cranmer Terrace, London, SW17 ORE, UK *Author for correspondence: sasharma@sgul.ac.uk

\section{KEYWORDS}

- athlete's heart • female $\bullet$ left ventricular geometry $\bullet$ physiological adaptation to exercise
"Although there is only a single chromosome separating the two sexes, females differ from males in several respects...all of which play a role in their cardiovascular response to athletic training." 
"Females exhibit qualitatively similar cardiac adaptation compared to males but show smaller dimensions." and symmetrical increase in right ventricular dimensions [9], however, female athletes exhibit smaller absolute values in all cardiac dimensions compared to male athletes [10-12]. Determinants of cardiac size include age, sex, ethnicity and sporting discipline [13]. Adult endurance athletes exhibit the largest dimensions $[1,14]$ and black athletes reveal a greater increase in LV wall thickness compared to athletes of other ethnicities $[15,16]$.

Some athletes, particularly those engaged in endurance sports, such as long distance running, cycling, swimming, rowing or canoeing, reveal large cardiac dimensions that overlap with those observed in patients with cardiomyopathy [1,14]. In such circumstance the differentiation between physiological adaptation and cardiac pathology is crucial because an erroneous diagnosis has serious consequences. An enlarged left ventricle consistent with dilated cardiomyopathy (DCM) is relatively common in males and is present in $14 \%$ of athletes. A wall thickness consistent with hypertrophic cardiomyopathy (HCM) is less common and is present in $2 \%$ of white males [1] and $13 \%$ of black males [15]. By contrast, few (1\%) female athletes show dimensions consistent with DCM and none reveal a LV wall thickness consistent with HCM [14]. These observations have important clinical implications, because an LV cavity exceeding $60 \mathrm{~mm}$ or a LV wall thickness greater than $12 \mathrm{~mm}$ should be viewed with suspicion and result further investigation to exclude cardiac pathology.

\section{LV geometry}

Although it is recognized that absolute cardiac dimensions are greater in male athletes compared to female athletes, sex differences in LV geometry have not been assessed in a large cohort of athletes. LV geometry takes into account the LV mass and relative LV wall thickness (ratio of LV wall thickness to LV cavity size). Normal geometry is defined as a normal LV mass and a relative wall thickness $<0.42$, concentric remodeling is defined as a normal LV mass and increased relative wall thickness $(>0.42)$, concentric LV hypertrophy is defined as an increased LV mass and a relative wall thickness and eccentric LV hypertrophy is defined as an increased LV mass and normal relative wall thickness [17]. We evaluated LV geometry in 1083 highly trained athletes, including 443 females in relation to sporting discipline. We observed two important findings. First, we noted that although females revealed smaller cardiac dimensions, they had a larger LV cavity compared to males when indexed for body surface area. Second, the same proportion of athletes (30\%) showed abnormal geometry but concentric LV hypertrophy was almost solely confined to males, whereas females engaging in endurance sports showed a higher prevalence of eccentric LVH compared to males. These results have important clinical implications because they indicate that the female athlete's heart adapts primarily by an increase in LV cavity size and concentric LV hypertrophy in females may be consistent with pathological LV hypertrophy [18]. None of the female athletes revealed a relative $L V$ wall thickness $>0.48$, therefore, this particular index could be used as a marker of abnormal LVH rather than absolute LV wall thickness measurements. Apart from a higher circulating concentration of testosterone and a higher density of myocardial testosterone receptors in males, a higher peak exercise related systolic blood pressure may also play an important role in the development of LV hypertrophy in males [19].

\section{Female athlete's ECG}

The athlete's ECG reflects vagotonia and increased cardiac chamber dimensions. As with cardiac dimensions, females reveal quantitatively smaller changes compared to males. PR interval and QRS duration are shorter and there is a considerably lower prevalence of voltage criteria for LV hypertrophy compared to males [20]. T-wave inversion in the lateral leads is exceptional in females and should raise the possibility of pathological LV hypertrophy. By contrast, females exhibit a slightly longer QT interval and a higher prevalence of $\mathrm{T}$-wave inversion in leads V1-V3 [21].

\section{Sudden death in sport}

Sudden cardiac death (SCD) in sport from a previously quiescent cardiac disease is fortunately rare but females are at a considerably lower risk than males. In competitive young athletes, the male to female ratio for sudden cardiac death is 10:1 [22], whereas in recreational sportsmen, this ratio increases to 20:1 [23]. These statistics are only partly explained by a relatively lower participation rate in women. It is possible that males ignore warning symptoms during exercise or attribute them to lack of fitness. There is also a possibility that high concentrations of androgens in males may accelerate the disease 
phenotype with cardiomyopathy [24] and atherosclerotic coronary artery disease, which are the most common causes of SCD in sport. In our own analysis of 357 consecutive deaths during exercise, we reported that $92 \%$ occurred in males [25]. Of the 27 deaths in females, the majority $(53 \%)$ showed a structurally normal heart indicative of an electrical fault, such as long QT syndrome, Brugada syndrome or catecholaminergic polymorphic ventricular tachycardia rather than cardiomyopathy or coronary artery disease.

\section{Veteran female athletes}

Over the past three decades there has been a surge in the number of veteran athletes (aged 40 years and above) engaging in endurance sports, such as marathons. The proportion of female athletes in this age category has grown relatively more than in males in the last 10 years. In parallel, there has been emerging evidence that athletes who have participated in lifelong exercise show an increased prevalence of atrial fibrillation [26,27], myocardial fibrosis and increased coronary artery calcification [28]. These reports suggest that excessive life-long endurance exercise may be deleterious for an otherwise normal heart in some individuals. Probably, the best evidence for this is the five-fold increase in prevalence of atrial fibrillation in veteran athletes compared to relatively sedentary individuals of similar age. However, the increased prevalence of atrial fibrillation appears to be confined to males only. We have just completed a study of 170 veteran athletes aged $54 \pm 8.5$ years $(29 \%$ female) and 132 controls who were of similar age, sex and had a low-risk score for coronary artery disease. We observed a higher prevalence of coronary plaques, myocardial fibrosis, atrial fibrillation and ventricular tachycardia in the

\section{References}

1 Pelliccia A, Maron BJ, Spataro A, Proschan MA, Spirito P. The upper limit of physiologic cardiac hypertrophy in highly trained elite athletes. N. Engl. J. Med. 324(5), 295-301 (1991).

2 Sharma S, Merghani A, Mont L. Exercise and the heart: the good, the bad, and the ugly. Eur. Heart J. 36(23), 1445-1453 (2015).

3 Zaidi A, Sharma S. Exercise and heart disease: from athletes and arrhythmias to hypertrophic cardiomyopathy and congenital heart disease. Future Cardiol. 9(1), 119-136 (2013).

athletes compared to the healthy controls but this phenomenon was limited to male athletes only [29]. It is plausible that if female athletes are less prone to sudden death from cardiac disease, their genetic and hormonal constitution may retard the shift from cardiac plasticity to arrhythmogenic remodeling.

\section{Conclusion \& future perspective}

In an era where the number of females participating in endurance events is increasing at a phenomenal rate, it is prudent to have some understanding of the impact of intensive exercise on the female heart. Females exhibit qualitatively similar cardiac adaptation compared to males but show smaller dimensions. It appears that endurance sportswomen adapt primarily by increasing ventricular dimensions as opposed to a marked increase in left ventricular wall thickness. Female athletes seem more protected than males from SCD during sport and the potentially deleterious effects of lifelong exercise on the heart. The large population of female athletes provides an opportunity for large and longitudinal studies to examine these sex differences and to develop a concrete understanding of the molecular mechanisms underlying these observations.

\section{Financial \& competing interests disclosure}

The authors have no relevant affiliations or financial involvement with any organization or entity with a financial interest in or financial conflict with the subject matter or materials discussed in the manuscript. This includes employment, consultancies, honoraria, stock ownership or options, expert testimony, grants or patents received or pending, or royalties.

No writing assistance was utilized in the production of this manuscript.

4 International Olympic Committee. Factsheet: Women in the Olympic Movement - Update May 2014. International Olympic Committee, Lausanne, Switzerland (2014).

5 Whyte GP, George K, Nevill A, Shave R, Sharma S, McKenna WJ. Left ventricular morphology and function in female athletes: a meta-analysis. Int. J. Sports Med. 25(5), 380-383 (2004).

6 McGill HC Jr, Sheridan PJ. Nuclear uptake of sex steroid hormones in the cardiovascular system of the baboon. Circ. Res. 48(2), 238-244 (1981).

7 Wernstedt P, Sjöstedt C, Ekman I et al. Adaptation of cardiac morphology and function to endurance and strength training. A comparative study using MR imaging and echocardiography in males and females. Scand. J. Med. Sci. Sports 12(1), 17-25 (2002).

8 Hutchinson PL, Cureton KJ, Outz H, Wilson G. Relationship of cardiac size to maximal oxygen uptake and body size in men and women. Int. J. Sports Med. 12(4), 369-373 (1991).

9 Henriksen E, Landelius J, Kangro T et al. An echocardiographic study of right and left ventricular adaptation to physical exercise in elite female orienteers. Eur. Heart J. 20 (4), 309-316 (1999). 
10 Pelliccia A, Maron BJ, Culasso F, Spataro A, Caselli G. Athlete's heart in women. Echocardiographic characterization of highly trained elite female athletes. JAMA 276(3), 211-215 (1996).

11 Rawlins J, Carre F, Kervio G et al. Ethnic differences in physiological cardiac adaptation to intense physical exercise in highly trained female athletes. Circulation 121(9), 1078-1085 (2010).

12 Whyte GP, George K, Nevill A, Shave R, Sharma S, McKenna WJ. Left ventricular morphology and function in female athletes: a meta-analysis. Int. J. Sports Med. 25(5), 380-383 (2004).

13 Zaidi A, Sharma S. Exercise and heart disease: from athletes and arrhythmias to hypertrophic cardiomyopathy and congenital heart disease. Future Cardiol. 9(1), 119-136 (2013).

14 Pelliccia A, Maron BJ, Culasso F, Spataro A, Caselli G. Athlete's heart in women. Echocardiographic characterization of highly trained elite female athletes. JAMA 276(3), 211-215 (1996).

15 Papadakis M, Carre F, Kervio G et al. The prevalence, distribution, and clinical outcomes of electrocardiographic repolarization patterns in male athletes of African/Afro-Caribbean origin. Eur. Heart J. 32(18), 2304-2313 (2011).

16 Rawlins J, Carre F, Kervio Get al. Ethnic differences in physiological cardiac adaptation to intense physical exercise in highly trained female athletes. Circulation 121(9), 1078-1085 (2010).

17 Lang RM, Bierig M, Devereux RB et al. Recommendations for chamber quantification. Eur. J. Echocardiogr. 7(2), 79-108 (2006).

18 Finocchiaro G, Papadakis M, Sharma S et al. Effect of gender and sporting discipline on left ventricular adaptation to exercise. Eur. Heart J. 36(Suppl. 1) (2015).

19 Zemva A, Rogel P. Gender differences in athlete's heart: association with 24-h blood pressure. A study of pairs in sport dancing. Int. J. Cardiol. 77(1), 49-54 (2001).

20 Storstein L, Bjørnstad H, Hals O, Meen HD. Electrocardiographic findings according to sex in athletes and controls. Cardiology 79(3), 227-236 (1991).

21 Malhotra A, Dhutia H, Gati S et al. 103 prevalence and significance of anterior $\mathrm{T}$ wave inversion in females. Heart 100 (Suppl. 3), A60-A60 (2014).

22 Maron BJ, Doerer JJ, Haas TS, Tierney DM, Mueller FO. Sudden deaths in young competitive athletes analysis of 1866 deaths in the United States, 1980-2006. Circulation 119(8), 1085-1092 (2009).

23 Marijon E, Tafflet M, Celermajer DS et al. Sports-related sudden death in the general population. Circulation 124(6), 672-681 (2011).
24 Olivotto I, Maron MS, Adabag AS et al. Gender-related differences in the clinical presentation and outcome of hypertrophic cardiomyopathy. J. Am. Coll. Cardiol. 46(3), 480-487 (2005)

25 Finocchiaro G, Papadakis M, Sharma S et al. Sudden death in sport: insights from a national pathology referral center. Eur. Heart J. 36(Suppl. 1) (2015).

26 Mont L, Tamborero D, Elosua R et al. Physical activity, height, and left atrial size are independent risk factors for lone atrial fibrillation in middle-aged healthy individuals. Europace 10(1), 15-20 (2008).

27 Mozaffarian D, Furberg CD, Psaty BM, Siscovick D. Physical activity and incidence of atrial fibrillation in older adults the cardiovascular health study. Circulation 118(8), 800-807 (2008).

28 Möhlenkamp S, Lehmann N, Breuckmann F et al. Running: the risk of coronary events: prevalence and prognostic relevance of coronary atherosclerosis in marathon runners. Eur. Heart J. 29(15), 1903-1910 (2008).

29 Merghani A, Alfakih K, Sharma S et al. The relationship between the dose of exercise and coronary artery calcification in veteran athletes. Eur. Heart J. 36(Suppl. 1) (2015). 